\title{
Production of Inhibitors of Lytic Activity in the Micrococcaceae
}

\author{
By PIETRO E. VARALDO,* FRANCESCA BIAVASCO AND \\ SEBASTIANO VALISENA \\ Institute of Microbiology, University of Genoa Medical School, Viale Benedetto XV, 10, \\ 16132 Genoa, Italy
}

(Received 21 April 1981; revised 7 July 1981)

\begin{abstract}
Eight Staphylococcus xylosus strains lacking lytic activity (LA) were found to excrete agar-diffusible factors inhibiting the LA of LA-positive strains of the same species. The same eight strains, when tested against LA-positive indicator strains from other species of Micrococcaceae, caused marked LA inhibition only of strains of $S$. saprophyticus and $S$. cohnii, both species closely related to $S$. xylosus. Micrococci and planococci, unlike staphylococci, do not normally show LA, yet Micrococcus lylae and M. sedentarius strains produced inhibitors effective against the LA of almost all the indicator strains, and planococci inhibited the LA of a few indicator strains. Micrococcus luteus strains caused LA inhibition of homologous indicators only, i.e. exceptional $M$. luteus strains exhibiting LA. Thus, a specific and a non-specific type of LA inhibition can be distinguished, and it is suggested that the apparent lack of LA might, in some instances, be explained by the simultaneous production of a specific inhibitor.
\end{abstract}

\section{INTRODUCTION}

A recent approach to the taxonomy and identification of the Micrococcaceae is based on the lytic activity (LA) of the strains. Six groups (lyogroups), closely related to species, were recognized within human staphylococci based on the analysis of their LA (Varaldo et al., $1978 a, b$; Varaldo \& Satta, 1978). Moreover, LA was shown to be a general feature of the genus Staphylococcus, whereas other Micrococcaceae (Micrococcus and Planococcus) lacked this property (Satta et al., 1977, 1978). Exceptions to this rule were, however, shown in previous studies, where both LA-negative staphylococci (under $0.5 \%$ of total staphylococci) and LA-positive micrococci (about $1 \%$ of total micrococci) were described (Satta et al., 1978; Varaldo et al., 1978a, 1979, 1980, 1981). Both exceptions were confined to specific groups of organisms, in particular $S$. xylosus for LA-negative staphylococci and $M$. luteus for LA-positive micrococci. The exceptional strains from both groups represent a scant minority within their respective species, since most $S$. xylosus strains are LA-positive, and belong to lyogroup IV (Varaldo \& Satta, 1978), while most $M$. luteus strains are LA-negative (Satta et al., 1978; Varaldo et al., 1979).

The present study shows that LA-negative $S$. xylosus strains excreted factors which inhibited the LA of LA-positive $S$. xylosus strains, suggesting that in the former, LA might be masked in vivo by such inhibitors. Extension of the study to micrococci and planococci showed that a specific and a non-specific type of LA inhibition existed: the former was effective with the LA of strains from homologous species, the latter with strains from different species.

\section{METHODS}

Strains tested for production of LA-inhibiting factors. Thirty LA-negative strains were tested. Eight were isolated in our laboratory from healthy human skin and identified as S. xylosus (Schleifer \& Kloos, 1975). 
Table 1. List of strains used (from the genera Staphylococcus, Micrococcus and Planococcus)

\section{Strains tested}

S. xylosus CM-20*

S. xylosus $\mathrm{CN}-11^{*}$

S. xylosus CO-07*

S. xylosus CO-13*

S. xylosus CP-14*

S. xylosus K2-12*

S. xylosus K3-04*

S. xylosus K3-12*

M. agilis CCM 2131

$M$. agilis CCM 2390

\section{S. aureus ATCC 12600}

S. aureus CCM 2504

S. aureus AT-12*

S. capitis CCM 2735

S. capitis AP-07*

S. cohnii CCM 2726

S. cohnii CCM 2736

S. cohnii BO-20*

S. epidermidis ATCC 14852

S. epidermidis ATCC 14990

$S$. epidermidis AH-15*

S. haemolyticus CCM 1798

S. haemolyticus CCM 2737

S. hominis CCM 2732

M. kristinae CCM 2690
M. kristinae CCM 2691
M. luteus ATCC 4698
M. luteus CCM 337
M. luteus CCM 351
M. luteus CCM 410
M. lylae CCM 2693
M. lylae CCM 2694
M. nishinomiyaensis CCM 2140
M. nishinomiyaensis CCM 2669

\section{Indicator strains}
S. hominis CCM 2733
S. hyicus CCM 2368
$S$. hyicus $\mathrm{SH} 1 \dagger$
S. intermedius CCM 5739
S. intermedius G8†
S. saprophyticus CCM 2682
S. saprophyticus CCM 2728
S. saprophyticus $\mathrm{BO}-03^{*}$
S. sciuri ATCC 29062
S. sciuri ATCC 29070
S. simulans CCM 2705
S. simulans O-12*
S. warneri CCM 2445
S. warneri CCM 2730

\author{
M. roseus CCM 633 \\ $M$, roseus CCM 837 \\ $M$. sedentarius CCM 314 \\ M. sedentarius CCM 2697 \\ $M$. varians CCM 418 \\ M. varians CCM 884 \\ $P$. citreus CCM 2389 \\ $P$. citreus CCM 2415 \\ Planococcus sp. CCM 2104 \\ Planococcus sp. CCM 2414
}

* From our Institute collection. $\quad \dagger$ Obtained from Dr V. Hajek, Olomouc, Czechoslovakia.

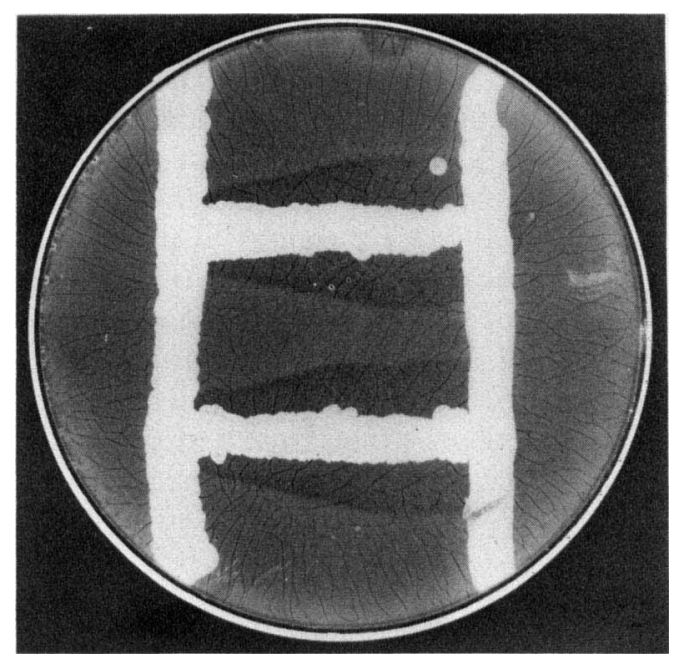

Fig. 1. Narrowing of the zones of transparency surrounding the streaks of two LA-positive $S$. xylosus indicators (strains CCM 1400 and CCM 2210, upper and lower horizontal streaks, respectively) caused by an LA-negative $S$. xylosus strain (CP-14, left vertical streak). No narrowing is caused by an LA-negative strain of $M$. varians (CCM 418, right vertical streak).

Eighteen strains were micrococci and four planococci (Table 1), all supplied by the Czechoslovak Collection of Microorganisms (CCM) or the American Type Culture Collection (ATCC).

Indicator strains. Forty-two LA-positive strains were used as indicators, i.e. their LA was the target of 
LA-inhibiting factors produced by the LA-negative test strains. These indicator strains included 38 staphylococci from 13 different species and four LA-positive $M$. luteus strains (Table 1).

Evaluation of $L A$ inhibition. Suspensions of the strains to be tested for production of LA inhibitors were made in sterile saline from $24 \mathrm{~h}$ cultures, and their turbidity was adjusted to about half that of a McFarland nephelometer barium sulphate no. 1 standard. Using a calibrated loop, standard amounts $(0.05 \mathrm{ml})$ of these suspensions were streaked on the surface of $15 \mathrm{~cm}$ diameter plastic dishes containing the LA test medium (AH47TP2, see Varaldo et al., 1978a, 1979, for its composition and preparation), making two parallel streaks of different strains about $9 \mathrm{~cm}$ apart. After incubation of the plates, which varied according to the growth rates of the strains tested, equal amounts of suspensions of indicator strains were streaked on the plate surface between and perpendicular to the two initial parallel streaks. Two to four different indicators were thus streaked on each plate so that their ends were close to the two streaks of the LA-negative test strains (Fig. 1). Plates were incubated for $48 \mathrm{~h}$ at $35^{\circ} \mathrm{C}$, and examined for zones of transparency around the indicator strains. A narrowing of this zone of transparency in the area near the perpendicular streak of an LA-negative strain was interpreted as production by the latter of some factor(s) inhibiting the LA of the indicator strain. The degree of inhibition was scored qualitatively as indicated in Table 2.

\section{RESULTS}

\section{Production of $L A$ inhibitors by LA-negative strains of $S$. xylosus}

The eight LA-negative strains of $S$. xylosus (Table 1) were tested for their ability to produce diffusible factors which inhibited the LA from ten $S$. xylosus indicator strains (Table 1). The LA of each indicator strain was inhibited to varying degrees by all eight LA-negative strains tested. The narrowing of the zone of transparency of indicator strains was not associated with growth inhibition.

In order to evaluate whether the LA inhibition exerted by the eight LA-negative strains of S. xylosus towards LA-positive strains of the same species was specific, the same eight strains were tested against a set of indicator strains including at least two representatives of all other recognized staphylococcal species. Four LA-positive $M$. luteus strains were also included in this set of indicators. The results (Table 2) showed that the LA of strains of $S$. aureus, $S$. epidermidis, $S$. simulans, $S$. intermedius, $S$. hyicus and $M$. luteus was not inhibited by the eight LA-negative $S$. xylosus strains. A sporadic and weak inhibition was observed with indicator strains of $S$. hominis, $S$. haemolyticus, $S$. warneri, $S$. capitis and $S$. sciuri. In contrast, a marked LA inhibition was generally observed with indicator strains of $S$. saprophyticus and $S$. cohnii. LA inhibition was never associated with any growth inhibition.

\section{Production of LA inhibitors by micrococci and planococci}

The finding that certain staphylococci apparently devoid of LA produced LA inhibitors, led us to investigate whether the ability to release similar inhibitors was a property shared by micrococci and planococci, which, unlike staphylococci, are generally LA-negative (Satta et al., 1978). Eighteen micrococci and four planococci, including at least two representatives of the recognized species, were tested for the release of LA inhibitors against a set of 35 indicator strains. LA inhibition effects varied with the species of the strains tested (Table 3 ). Strains of $M$. varians, $M$. roseus, $M$. nishinomiyaensis, $M$. kristinae, and $M$. agilis produced virtually no inhibition of the LA of the indicator strains, whereas inhibition was sporadically exhibited by planococci. A strong inhibition effective against the LA of almost all the indicator strains was displayed by the strains of $M$. lylae and $M$. sedentarius. A more specific behaviour was shown by the four strains of $M$. luteus, which consistently caused inhibition of the LA of homologous indicator strains (i.e. LA-positive $M$. luteus strains), but caused virtually no inhibition of the LA of the other indicator strains. Again, the narrowing of the zones of transparency of indicator strains denoting LA inhibition was never associated with any growth inhibition.

\section{DISCUSSION}

The LA inhibition exerted by LA-negative strains towards LA-positive strains of Micrococcaceae, as shown in this study, seems to be mediated by extracellular agar-diffusible 


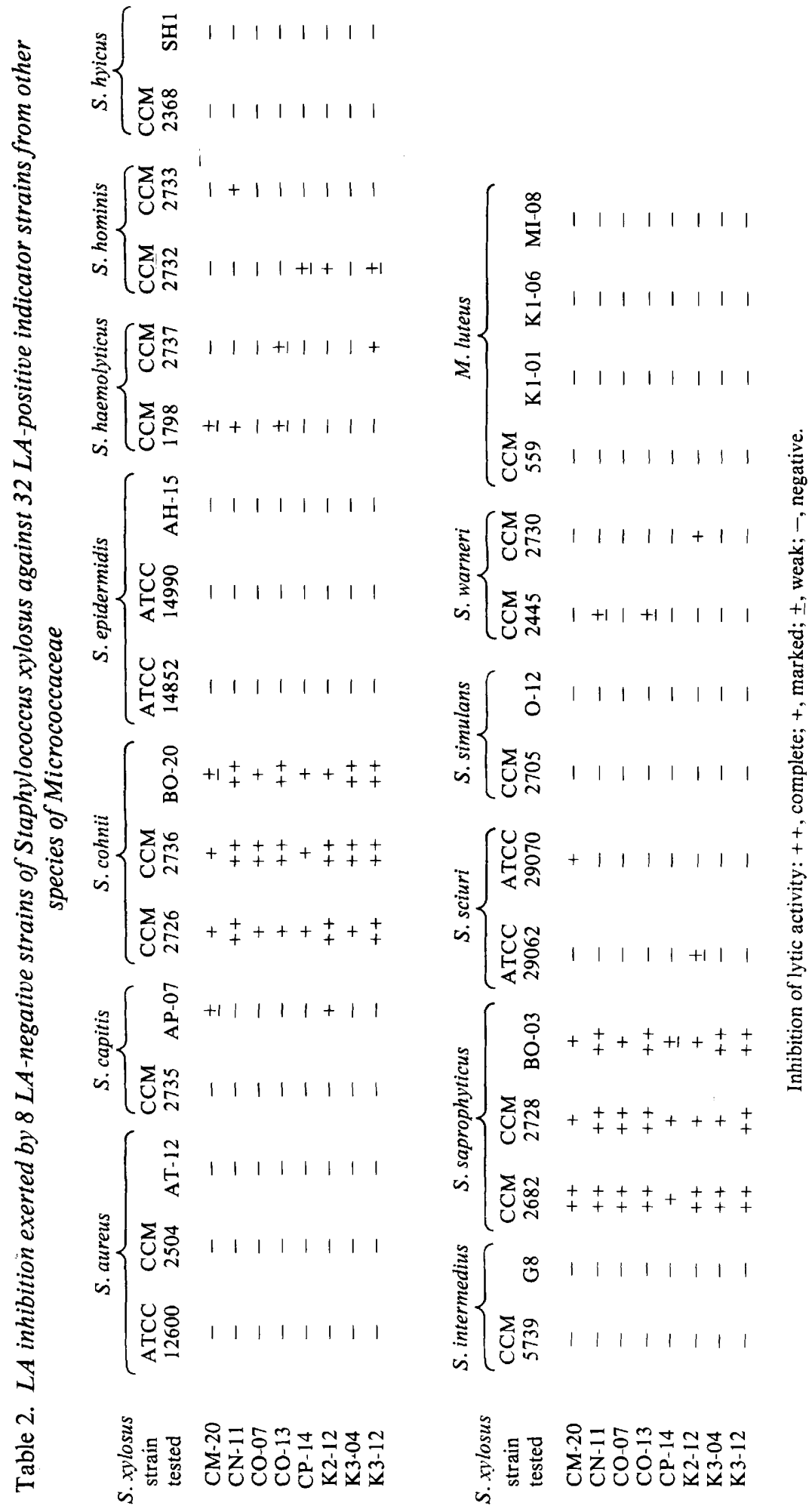




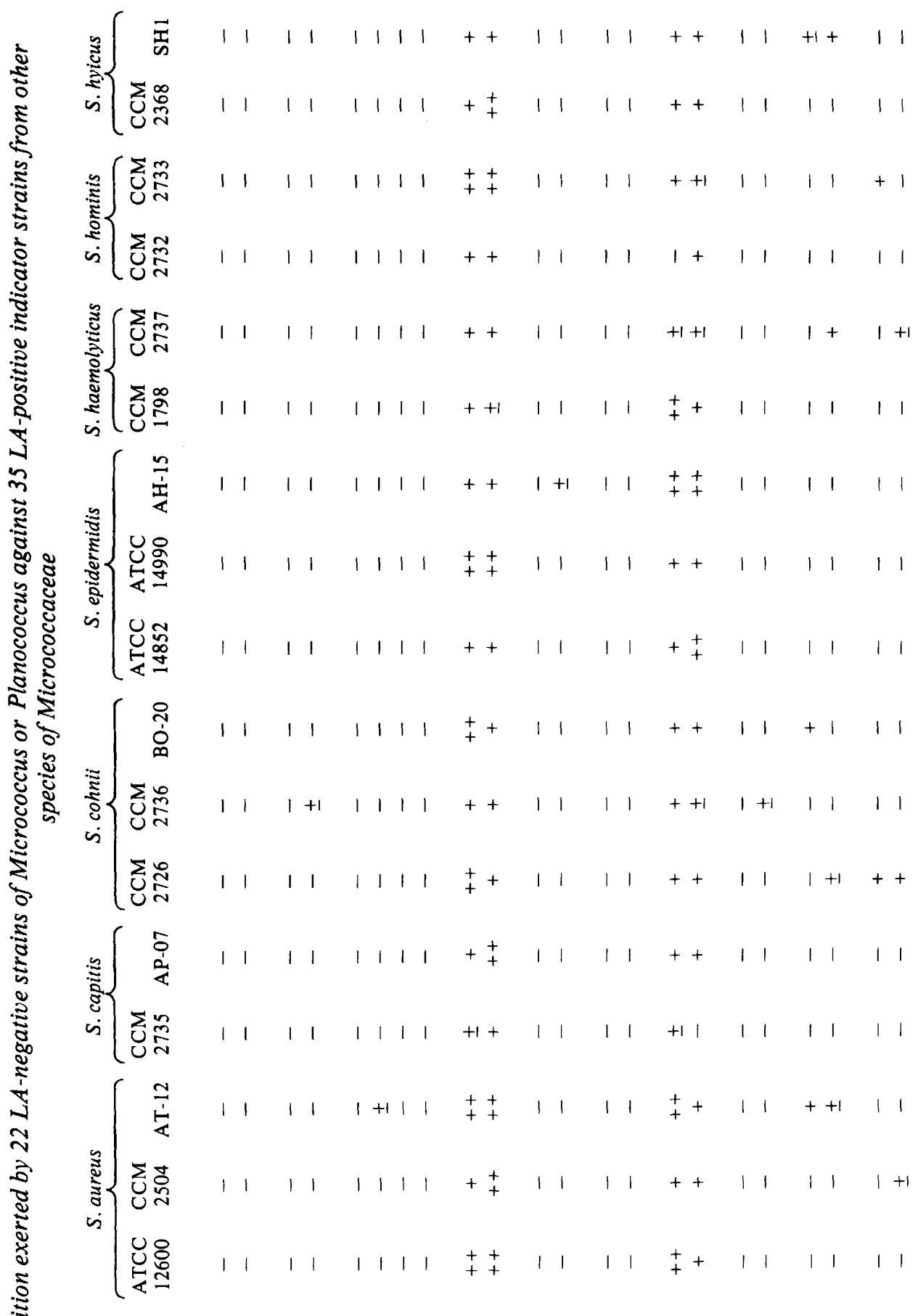

衤

峞

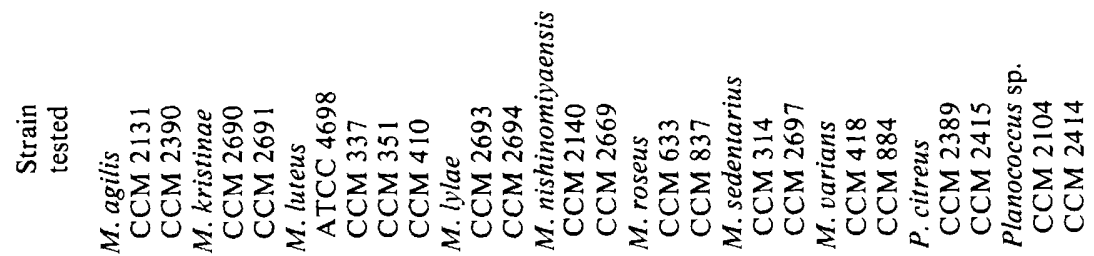




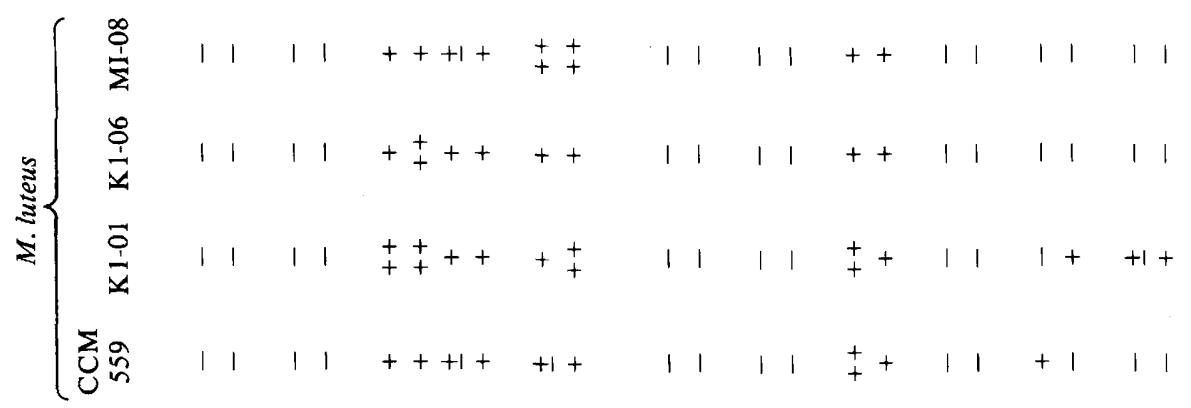

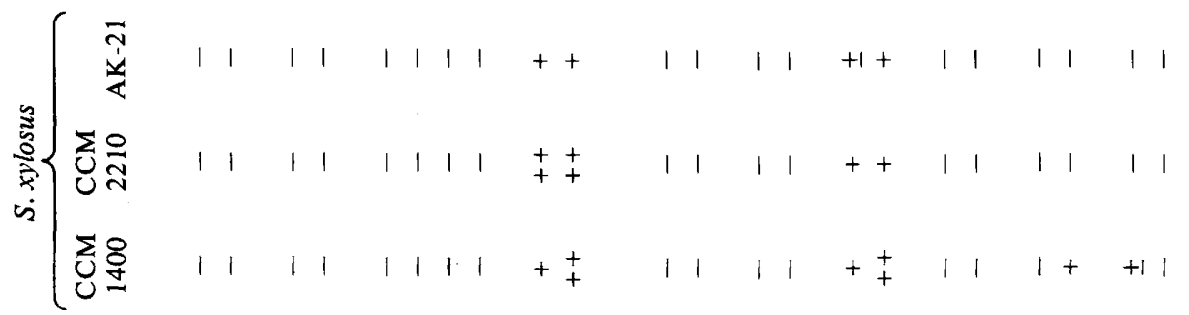

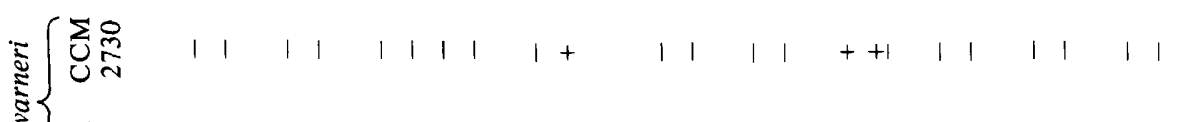

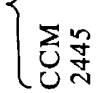

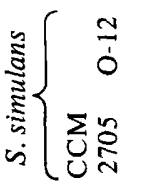

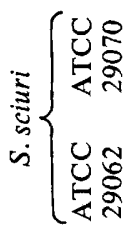

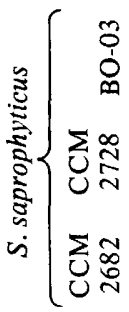

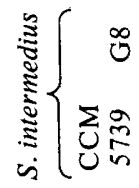


factors. This is suggested by the experimental conditions under which LA inhibition has been detected and by the complete and consistent absence of any growth reduction associated with LA inhibition. We have recently isolated a fraction which is resistant to proteolysis from the culture supernatant of a LA-negative $S$. xylosus strain. This compound inhibits the LA from LA-positive strains of the same species. Further investigation is underway in our laboratory to define the chemical nature as well as the physiological significance of these inhibitors of lytic enzymes. These enzymes are known to be required for cell wall extension and division (Rogers, 1970; Shockman et al., 1974) and inhibitors have been described recently and shown, in some instances, to play a role in the regulation of cell wall biosynthesis (Ghuysen, 1977).

Our findings suggest that a specific and a non-specific type of LA inhibition may be distinguished. The latter seems to be peculiar to $M$. lylae and $M$. sedentarius strains which caused strong LA inhibition when tested against indicator strains from all LA-positive Micrococcaceae examined. Similarly, LA inhibition by planococci was non-specific, but less marked and consistent than that caused by $M$. lylae and $M$. sedentarius. In contrast, specific LA inhibition was displayed by $M$. luteus strains, which released factors effective against the LA of LA-positive strains of the same species only. Similarly, the LA inhibition caused by LA-negative $S$. xylosus, although apparent against indicators from three species ( $S$. xylosus, $S$. saprophyticus and $S$. cohnii), should be regarded as specific. The species $S$. saprophyticus, $S$. cohnii and $S$. xylosus are in the same lyogroup (Varaldo \& Satta, 1978); consequently both LA-negative staphylococci, and those LA-positive staphylococci undergoing inhibition of their LA caused by the former, belong to a well-defined three-species group. Moreover, these species are known to be very closely related in other respects including low acid production from glucose under anaerobic conditions, resistance to novobiocin, and DNA homology (Schleifer \& Kloos, 1975; Schleifer et al., 1979). It is consistent that strains from species which are closely related and share the same type of LA should also share susceptibility to a particular LA inhibitor.

Differences from species to species in the production of or susceptibility to LA inhibitors, together with the established taxonomic relevance of LA within Micrococcaceae (Varaldo et al., 1978a, b; Varaldo \& Satta, 1978; Satta et al., 1978), suggest that further studies of LA inhibitors may be useful for taxonomic purposes.

Within $S$. xylosus and $M$. luteus (the species containing LA-negative strains which produced specific LA inhibitors), the lack of observable LA by a given strain might reflect its ability to produce the specific inhibitor rather than its failure to produce the lytic enzymes. Thus, the LA would be masked by the specific inhibitor and the strain would appear LA-negative. The lack of LA in certain staphylococci (a minority of $S$. xylosus strains) might be due to their ability to produce a specific LA inhibitor. Conversely, the exceptional LA-positive micrococci (a minority of $M$. luteus strains) might result from the loss of a specific LA inhibitor normally produced by strains of this species. As LA has not so far been observed within other micrococcal or planococcal species (Satta et al., 1978), a specific LA inhibition cannot be observed in these instances. However, from the above hypothesis, it may be that simultaneous production of specific LA inhibitors is the reason for the apparent failure of these strains to produce LA.

The authors wish to thank Dr Giuseppe Satta for helpful discussions and comments on the manuscript.

\section{REFERENCES}

GHuysen, J. M. (1977). Biosynthesis and assembly of bacterial cell walls. In The Synthesis, Assembly and Turnover of Cell Surface Components, Cell Surface Reviews, vol. 4, pp. 463-595. Edited by G. Poste \& G. L. Nicolson. Amsterdam: North-Holland.
Rogers, H. J. (1970). Bacterial growth and the cell envelope. Bacteriological Reviews 34, 194-214.

Satta, G., Varaldo, P. E., Grazi, G. \& Fontana, R. (1977). Bacteriolytic activity in staphylococci. Infection and Immunity 16, 37-42. 
Satta, G., Varaldo, P. E., Tenca, M. \& Radin, L. (1978). The relevance of bacterial lytic activity in the taxonomy of the Micrococcaceae: failure of its production by Micrococcus and Planococcus as opposed to Staphylococcus. Journal of General Microbiology 109, 385-388.

SCHLEIFER, K. H. \& KLoos, W. E. (1975). Isolation and characterization of staphylococci from human skin. I. Amended descriptions of Staphylococcus epidermidis and Staphylococcus saprophyticus, and descriptions of three new species: Staphylococcus cohnii, Staphylococcus haemolyticus, and Staphylococcus xylosus. International Journal of Systematic Bacteriology 25, 50-61.

SCHLEIFER, K. H., MeYER, S. A. \& Rupprecht, M. (1979). Relatedness among coagulase-negative staphylococci: deoxyribonucleic acid reassociation and comparative immunological studies. Archives of Microbiology 122, 93-101.

Shockman, G. D., Daneo-Moore, L. \& Higgins, M. L. (1974). Problems of cell wall and membrane growth, enlargement and division. Annals of the New York Academy of Sciences 235, 161-197.

VARALDo, P. E. \& SATTA, G. (1978). Grouping of staphylococci on the basis of their bacteriolyticactivity patterns: a new approach to the taxonomy of the Micrococcaceae. II. Main characters of 1,054 strains subdivided into 'lyogroups'. International Journal of Systematic Bacteriology 28, 148-153.
Varaldo, P. E., Satta, G., Grazi, G. \& Romanzi, C. A. $(1978 a)$. Grouping of staphylococci on the basis of their bacteriolytic-activity patterns: a new approach to the taxonomy of the Micrococcaceae. I. Identification of six different 'lyogroups'. International Journal of Systematic Bacteriology 28, 141-147.

VARAldo, P. E., Satta, G. \& HäJek, V. (1978b). Taxonomic study of coagulase-positive staphylococci: bacteriolytic activity pattern analysis. International Journal of Systematic Bacteriology 28, 445-448.

Varaldo, P. E., Grazi, G., Cisani, G. \& Satta, G. (1979). Routine separation of staphylococci from micrococci based on bacteriolytic activity production. Journal of Clinical Microbiology 9, 147-148.

Varaldo, P. E., Grazi, G., Soro, O., Cisani, G. \& SAtTA, G. (1980). Simplified lyogroup system, a new method for routine identification of staphylococci: description and comparison with three other methods. Journal of Clinical Microbiology 12, 63-68.

VAraldo, P. E., Soro, O., Grazi, G. \& Biavasco, F. (1981). Clinical distribution and antibiotic sensitivities of staphylococcal strains isolated over an eight-month period. Journal of Clinical Pathology 34, 443-447. 